TTR

Traduction, terminologie, rédaction

\title{
Toucher les souffles marginaux : réflexions sur quelques reflets de la banlieue parisienne au Japon
}

\section{Satoshi Udo}

Volume 31, numéro 1, 1er semestre 2018

Traduire la banlieue : problématiques, enjeux, perspectives Translating the Banlieue: Issues, Challenges, Perspectives

URI : https://id.erudit.org/iderudit/1062551ar

DOI : https://doi.org/10.7202/1062551ar

Aller au sommaire du numéro

\section{Éditeur(s)}

Association canadienne de traductologie

ISSN

0835-8443 (imprimé)

1708-2188 (numérique)

Découvrir la revue

\section{Citer cet article}

Udo, S. (2018). Toucher les souffles marginaux : réflexions sur quelques reflets de la banlieue parisienne au Japon. TTR, 31(1), 171-192.

https://doi.org/10.7202/1062551ar

\section{Résumé de l'article}

Cet article vise à montrer comment les Japonais ont observé la banlieue de France au cours des trente dernières années. Il vise également à examiner comment ils ont « traduit » la banlieue, c'est-à-dire comment ils l'ont rendue compréhensible au-delà de sa surface médiatisée, en recourant à la littérature. La littérature japonaise a découvert de nouvelles valeurs dans la banlieue de Tokyo à travers la traduction des littératures occidentales : la banlieue représente un lieu de promenade inspiré par Tourgueniev et basé sur une nouvelle sensibilité envers la nature, ou encore le lieu où les enfants de la classe moyenne sont entourés de soins familiaux, comme le raconte la littérature anglaise pour enfants. Les traductrices japonaises de la littérature de banlieue française tentent de présenter les souffles des personnes qui y vivent, que les recherches scientifiques ou les médias ne sauraient saisir, mais elles ne peuvent s'empêcher, par ailleurs, d'insérer des notes dans leurs traductions pour aider les lecteurs à comprendre le contexte sociologique des oeuvres. À titre d'exemple, Vers la banlieue (1995) d'Horie Toshiyuki est conçu, selon l'auteur, pour représenter la " position » de la banlieue parisienne, tandis que la traduction du roman Le petit Malik de Mabrouck Rachedi (2008) est réalisée pour transmettre les voix de la banlieue. Rachedi aborde pleinement les conditions socioculturelles de la banlieue dans son ouvrage et cette tendance est accentuée par l'apparat de notes dans la traduction. Horie, en revanche, laisse de côté la situation sociologique en traduisant la banlieue de Paris dans les impressions de paysages procurées par les détours livresques. La banlieue reste ainsi un lieu en devenir, où les promeneurs s'égarent en rêveries, et la traduction est un lieu de partage où le sens est suspendu et remis en question.
Ce document est protégé par la loi sur le droit d'auteur. L’utilisation des services d'Érudit (y compris la reproduction) est assujettie à sa politique d'utilisation que vous pouvez consulter en ligne.

https://apropos.erudit.org/fr/usagers/politique-dutilisation/ 


\title{
Toucher les souffles marginaux : réflexions sur quelques reflets de la banlieue parisienne au Japon
}

\author{
Satoshi Udo \\ Université de Kagoshima
}

\section{Résumé}

Cet article vise à montrer comment les Japonais ont observé la banlieue de France au cours des trente dernières années. Il vise également à examiner comment ils ont «traduit» la banlieue, c'est-à-dire comment ils l'ont rendue compréhensible au-delà de sa surface médiatisée, en recourant à la littérature. La littérature japonaise a découvert de nouvelles valeurs dans la banlieue de Tokyo à travers la traduction des littératures occidentales : la banlieue représente un lieu de promenade inspiré par Tourgueniev et basé sur une nouvelle sensibilité envers la nature, ou encore le lieu où les enfants de la classe moyenne sont entourés de soins familiaux, comme le raconte la littérature anglaise pour enfants. Les traductrices japonaises de la littérature de banlieue française tentent de présenter les souffles des personnes qui $\mathrm{y}$ vivent, que les recherches scientifiques ou les médias ne sauraient saisir, mais elles ne peuvent s'empêcher, par ailleurs, d'insérer des notes dans leurs traductions pour aider les lecteurs à comprendre le contexte sociologique des œuvres. A titre d'exemple, Vers la banlieue (1995) d'Horie Toshiyuki est conçu, selon l'auteur, pour représenter la "position » de la banlieue parisienne, tandis que la traduction du roman Le petit Malik de Mabrouck Rachedi (2008) est réalisée pour transmettre les voix de la banlieue. Rachedi aborde pleinement les conditions socioculturelles de la banlieue dans son ouvrage et cette tendance est accentuée par l'apparat de notes dans la traduction. Horie, en revanche, laisse de côté la situation sociologique en traduisant la banlieue de Paris dans les impressions de paysages procurées par les détours livresques. La banlieue reste ainsi un lieu en devenir, où les promeneurs s'égarent en rêveries, et la traduction est un lieu de partage où le sens est suspendu et remis en question.

Mots-clés: banlieue de Paris, banlieue de Tokyo, immigrés, Mabrouck Rachedi, Horie Toshiyuki 


\begin{abstract}
This article aims to show how the Japanese have observed the suburbs of France for thirty years, and examine how they "translated" the suburbs, that is to say how they made it understandable beyond its reported surface, by resorting to literature. Japanese literature discovered new values in the suburbs of Tokyo brought by the translation of Western literatures: the wood-walk is inspired by Turgenev and based on a new sensitivity towards nature, and the middle-class children are surrounded by care in the family as told in English children's literature. Currently, Japanese translators of the French suburbs' literature are trying, on the one hand, to present the breath of people living there that scientific research as media cannot grasp, but they cannot shirk, on the other hand, to use notes to help readers understand the sociological context of the works. Towards the suburbs (1995) of Horie Toshiyuki is conceived, according to the author, to represent the "position" of the Parisian suburbs, while the translation of Le petit Malik by Mabrouck Rachedi (2008) is made to convey the voices of the suburbs. Rachedi fully brings the sociocultural conditions of the suburbs in his book and this trend is accentuated by the annotated translation. Horie, on the other hand, drops the sociological situation by translating the suburbs of Paris into the impressions of landscapes procured by the bookish detours. The suburb thus remains a place in the making, where the walkers are wandering in daydreams, and the translation is a common place where the meaning is suspended and questioned.
\end{abstract}

Keywords: suburbs of Paris, suburbs of Tokyo, immigrants, Mabrouck Rachedi, Horie Toshiyuki

\title{
Introduction
}

La France a été un pays de référence pour les Japonais, qui ne se sont jamais contentés d'imiter les systèmes anglo-américains pour envisager la modernisation de leur société. Les intellectuels francisants, surtout les sociologues mais aussi les littéraires, ont discuté, par exemple, de citoyenneté, de républicanisme, de laïcité, de communautarisme, d'intégration des immigrés ou des réfugiés. L'un des chercheurs les plus célèbres ayant étudié la société française et surtout sa banlieue est Miyajima Takashi ${ }^{1}$, professeur émérite à l'Université pour femmes d'Ochanomizu. À partir de 1985, il a publié au Japon les premières études sur la banlieue de Paris. Son dernier livre, intitulé Tabunka dearukoto toha [Ce quêtre multiculturel] (2014), ne se limite pas au lectorat universitaire, mais présente à un public japonais général certains des défis socioculturels auxquels le

1. Dans le présent article, tous les noms de famille japonais précèdent les prénoms, selon l'usage en japonais. 
pays est confronté et propose des solutions pour réaliser une société multiculturelle et pluraliste, en faisant référence au cas français. Les études socioculturelles menées en France ou portant sur la France sont ainsi devenues, dans un sens, un héritage intellectuel pour concevoir la société japonaise à venir.

Une sociologue de la génération suivante, Mori Chikako, professeure associée à l'Université d'Hitotsubashi, a récemment publié une édition augmentée et refondue de sa thèse, soutenue en 2010, sous le titre Haijo to teikô no kôgai [Exclusion et résistance dans les banlieues : les quartiers populaires des «immigrés» en France, formation et transformations] (2016). L'ouvrage a été couronné de deux prix prestigieux, le prix Osaragi Jirô de la critique et le prix ShibusawaClaudel, qui soulignent non seulement la qualité scientifique du travail de Mori, mais aussi l'intérêt que portent les intellectuels japonais à des sujets tels que l'intégration des immigrés. De fait, Mori amorce l'introduction de son livre par une référence aux attentats terroristes survenus dans les banlieues parisiennes en 2015, banlieues que les médias japonais ont présentées comme des zones pauvres et dangereuses, peuplées d'immigrés mal intégrés. Dès lors, pour les Japonais, les immigrés ou les réfugiés sont parfois considérés comme "dangereux», et les grandes villes européennes ne se distinguent plus de leurs banlieues, qui semblent «mises en péril». Les impressions (principalement négatives) suscitées par la banlieue parisienne sont à l'heure actuelle très vives et dérangeantes, ce qui finira par brouiller les idées et connaissances longtemps accumulées à leur égard. Nous voulons donc, dans le présent article, montrer de quelle manière les Japonais observent la banlieue de France depuis une trentaine d'années, pour examiner ensuite comment ils ont «traduit» la banlieue, c'est-à-dire comment ils l'ont rendue compréhensible ou plutôt palpable, au-delà de sa surface médiatisée, par le truchement de la littérature.

\section{La banlieue française au Japon}

C'est Hayashi Mizue qui, la première, a présenté la banlieue de Paris au public japonais. Après avoir mené de vastes recherches de terrain, la sociologue a rédigé un reportage intitulé Furansu no Ihôjin [Les étrangers à Paris], qui a été publié en 1984 en format poche chez Chûôôron-sha, un des plus célèbres éditeurs japonais, dont la collection de poche Chûkô Shinsho est présente dans la plupart des bibliothèques publiques, universitaires et lycéennes. Ce 
livre, fort abordable par son prix et son accessibilité de même qu'en raison de son style très lisible et analytique basé sur des connaissances approfondies, a été salué par la critique ainsi que par nombre de lecteurs. Bien que quelques reportages au sujet des immigrés en France aient été récemment publiés au Japon en format poche ${ }^{2}$, le livre d'Hayashi demeure la principale référence (recommandée entre autres aux étudiants) pour connaître la situation de la banlieue ainsi que celle des immigrés et de leurs droits en France. Certes, les données ont quelque peu vieilli, mais les problématiques majeures sont déjà décrites dans cet ouvrage. Chaque fois que nous le relisons, nous nous étonnons du fait que l'argumentation de l'auteure n'a pas pris une ride et que nos sociétés nont pas beaucoup avancé dans la résolution des problèmes relevés il y a plus de trente ans. La postface du livre montre l'intérêt que porte l'auteure aux différences des sociétés française et japonaise :

Pendant mon séjour en France, une fois, j'ai invité dans un restaurant japonais une dame française, une villageoise qui était passée à Paris. À la vue de l'intérieur du restaurant, elle m'a demandé ce qui suit : «Tous les Japonais ont les cheveux noirs?»

Trop accoutumés aux rapports entre des gens à la même couleur de cheveux, nous avons tendance, même lorsque nous regardons la France de loin, à imaginer qu'elle est également un pays très «homogène». On n'avait pas osé en parler autant jusqu'aujourd'hui, mais la société française est, en fait, un «creuset de races» qui rassemble de nombreuses ethnies à côté d'une majorité française. La «grande» culture française que les Japonais n'ont cessé d'admirer depuis l'époque de Meiji [1868-1912] est née de ce terreau.

Pourtant, récemment dans cette France, on a commencé à dire souvent, en se surveillant, qu'«il faut savoir que la France est une société multiethnique». Mais pourquoi? Dans ce livre j’ai essayé d'observer les circonstances derrière cela, en citant les exemples les plus récents et les plus concrets possible, en écoutant la voix des intéressés. Toutefois, à cause du visage trop complexe de chaque groupe cité, j'ai fini par entrevoir avec peine son profil. Tous ces thèmes, que j'ai traités dans différents chapitres, devraient être approfondis.

Il y a une décennie, les Français qui s'installaient dans un village de campagne étaient aussi des «intrus», c'est-à-dire des "étrangers»

2. Un de ces reportages, Imin to gendai furansu [Les immigrés et la France contemporaine] (2003) de Muriel Jolivet, professeure à l'Université de Sophia (Tokyo), aborde le roman Le Gone du Chaâba (1986) d'Azouz Begag. 
aux yeux des villageois. Les étrangers contemporains cités dans le présent livre représentent environ dix pour cent de toute la population française. Les spécificités de ce groupe réclament donc une nouvelle conception de la France en tant que société multiethnique. À cela s'ajoutent des problèmes graves et des perceptions de plus en plus négatives. Cependant, cela ne signifie pas que toutes les personnes qui appartiennent à ce dix pour cent souffrent des malaises décrits dans le présent livre.

Le plus important, c'est qu'aujourd'hui, en France, tout le monde, les politiciens comme les citoyens, malgré leurs difficultés face à des situations nouvelles, est en train de chercher sérieusement une voie de coexistence qui ne force pas l'assimilation, et continuent leurs luttes actives. (Hayashi, 1984, p. 218-219; notre trad.)

Au cours des trente dernières années, les Japonais se sont aperçus, enfin et petit à petit, que la société japonaise n'est pas aussi «homogène » que nous l'avions généralement conçue : il suffit, pour s'en convaincre, de considérer la présence d'une grande population coréenne qui remonte partiellement à l'époque coloniale ainsi que des autochtones d'Hokkaidô, les Aïnou, qui ont été assimilés aux Japonais depuis la formation de l'État-nation. En outre, le nombre d'ouvriers étrangers ne cesse d'augmenter tandis que le taux de naissance chute irrémédiablement. Pourtant, le chauvinisme attisé par certains internautes ainsi que l'ignorance sur les immigrés de facto mettent à dure épreuve la société japonaise, qui n'a pas encore relevé le défi de l'hétérogénéité qu'elle vient à peine de reconnaître.

Si l'on compare les deux ouvrages sociologiques, à savoir le reportage d'Hayashi et l'étude de Mori, on remarque que le terme «banlieue» a graduellement mûri dans la langue japonaise durant ces trente années, ce qui lui permet maintenant d'avoir deux principales connotations : la première fait référence aux zones peuplées par la classe moyenne autour de Tokyo et la seconde, à la banlieue «délicate» inspirée par celle de Paris.

Dans Furansu no Ihôjin [Les étrangers à Paris] de Hayashi Mizue (1984), le terme «banlieue» ne désigne que certains territoires purement géographiques, tandis que les termes «cité» et «bidonville» impliquent nécessairement des connotations symboliques caractérisant la vie des immigrés. En revanche, le livre de Mori Chikako, Haijo to teikô no kôgai [Exclusion et résistance dans les banlieues : les quartiers populaires des «immigrés» en France, formation et transformations] (2016), porte dans le titre le mot «banlieue» et commence par la présentation «des images de la banlieue [de 
France] qui diffère de celle du Japon»(p.11; notre trad.), là où l'auteure suppose que les Japonais associent la «banlieue » aux images des quartiers de la classe moyenne japonaise, comparables aux aires résidentielles anglo-américaines. Elle souligne que la «banlieue» de France a été présentée par les médias japonais comme un "ghetto» ou un "espace dangereux", en citant quelques articles de journaux publiés lors des «émeutes en France» de 2005, dont la violence a définitivement impressionné la société japonaise.

\section{La banlieue de Tokyo et ses représentations littéraires}

Tokyo, héritière de l'ancienne capitale du shogunat Edo divisée en deux territoires - celui des samouraïs aristocrates et celui des gens du peuple - est restée longtemps, même après la Restauration du Meiji (1868), composée de deux parties: Yamanote [côté de collines], les quartiers hauts où vivent les résidents aisés, et Shitamachi [ville basse], où se trouvent les quartiers populaires. La banlieue de Tokyo, en fait, s'est formée sur l'étendue (vers l'ouest) de Yamanote comme un nouvel espace petit-bourgeois, tandis que Shitamachi a été conçu, surtout en littérature, comme un quartier périssant qui préserve le passé. Plusieurs grandes figures japonaises de la littérature moderne, parmi lesquelles Mori Ôgai (1862-1922) et Nagai Kafû (1879-1959), ont aimé et décrit dans leurs œuvres ces quartiers qui étaient en train de disparaître et, avec eux, les mémoires d'Edo qu'ils conservaient.

La banlieue de Tokyo a ensuite été imaginée et décrite dans la littérature contemporaine. D'après Kawamoto Saburô, célèbre critique littéraire et auteur de Kôgai no bungakushi [L'bistoire littéraire de banlieue] (2003), la banlieue était autrefois une sorte de "frontière» (p. 36; notre trad.) pour les Américains et constituait par ailleurs l'espace idéal et idéalisé pour les familles de la petite bourgeoisie qui, en réalité, ont été poussées hors du centre-ville par la pression démographique et économique. La forte augmentation de la population dans la capitale, surtout dans les décennies 1920-1930, en raison de l'accroissement des salariés, a nécessité l'exploitation de terrains pour bâtir de nouvelles habitations ${ }^{3}$. Kawamoto conclut que la banlieue de Tokyo est «le lieu où "le bonheur modeste des petits-bourgeois" a été rêvé, enfanté, détruit et encore une fois rêvé,

3. Notons que le grand déplacement de la population vers la campagne, à l'ouest, a été causé également par la destruction totale de la ville par le séisme de Kantô en 1923 ainsi que par les bombardements américains en 1945. 
où la mort et la renaissance du rêve se sont répétées» (ibid., p. 22; notre trad.). C'est un espace de vie idéalisé par les jeunes familles, c'est là où la littérature pour enfants est née, sous l'influence de la littérature anglaise, avec ses figures d'«enfants entourés de soins affectueux» (ibid., p. 4; notre trad.) dans une banlieue alors pleine de verdure. Le roman pionnier du genre, Non-chan kumo ni noru [ La petite Non monte en nuages] (1951), écrit par Ishii Momoko (19072008), fondatrice et grande traductrice de littérature pour enfants au Japon, a été rédigé pendant la Deuxième Guerre mondiale en souvenir de la vie heureuse de son enfance dans la banlieue d'avantguerre. Cette vie de classe moyenne décrite dans les romans, les feuilletons télévisés et les mangas, a longtemps été perçue comme typique de la nation japonaise : nous pouvons en relever plusieurs exemples dans les œuvres de Mukôda Kuniko (1929-1981), écrivaine de feuilletons très populaire, et dans celles de la première auteure professionnelle de mangas, Hasegawa Machiko ${ }^{4}$ (19201992). De plus, le grand succès de Tonari no Totoro [Mon voisin Totoro] (1988), film d'animation de Miyazaki Hayao, atteste que cette «banlieue idéalisée » suscite encore une certaine nostalgie chez le public japonais des années 1980.

En fait, il importe de mentionner que la beauté de cette banlieue toute en verdure et de ses paysages d'un goût sauvage a été «découverte» à travers la traduction littéraire. En effet, le précurseur de cette nouvelle esthétique de la banlieue, Kunikida Doppo (1871-1908), a appris à observer et à apprécier la nature grâce à Mémoires d'un chasseur (1852) d'Ivan Tourgueniev, traduit en 1888 par Futabatei Shimei (1864-1909), auteur du premier roman moderne japonais, Ukigumo [Nuages flottants] (1887). Loriginalité de Doppo réside dans le fait qu'il a découvert la beauté agreste de la banlieue et a ainsi réalisé le récit Musashino [Champs de Musashi] (1901), observant les paysages innocents de la nature à côté de la grande ville de Tokyo. Le narrateur-auteur se promène le long de ruisseaux, pénètre dans les bois et, en rentrant à la maison, lit William Wordsworth ou Léon Tolstoï. Or, dans la première édition publiée en revue, le narrateur promène son «chien » (supprimé dans le livre), ce qui révèle, d'après Kawamoto, l'influence de l'ouvrage de Tourgueniev; en effet, la promenade dans les bois nécessite un

4. Son énorme best-seller Sazae-san (1946-1974), vendu à plus de 86 millions d'exemplaires en série et adapté en dessin animé, illustre depuis 1969 la vie quotidienne d'une famille ordinaire, sa propre famille. 
chien... c'est une formule littéraire (cf. Satô, 1917). Tayama Katai (1872-1930), fondateur du naturalisme japonais, se souvient d'avoir dégusté chez Doppo du curry et du café au lait (produits très rares à l'époque), ce qui montre que la banlieue était alors le nouveau lieu où la civilisation occidentale rencontrait la nature champêtre, dont la beauté venait d'être découverte à travers un nouveau prisme littéraire qui passait par la traduction.

La banlieue comme topos du «bonheur modeste des petitsbourgeois» a survécu jusqu'au début du $\mathrm{XX}^{\mathrm{e}}$ siècle, grâce entre autres à Shôno Junzô (1921-2009), qui, pendant un demi-siècle, n'a cessé d'écrire des autofictions autour de sa famille vivant dans la banlieue tokyoïte. Sa plume très «modeste», influencée par les essais de Charles Lamb et les nouvelles de Charles Philippe, se concentre sur les petites joies de la vie quotidienne qui, malgré son apparence paisible, n'est cependant plus aussi sûre qu'elle n'était auparavant (l'auteur le savait peut-être parce qu'il avait été recruté très jeune dans l'armée et avait été sauvé par la fin inattendue de la guerre). Shôno confie dans Zabon no hana [La fleur de pamplemoussier] (1956) sa décision de protéger sa nouvelle maison, sa détermination à s'enraciner dans la terre qui vient juste d'être défrichée, quoiqu'il faille du temps et de la patience. Disons que la banlieue de Tokyo a, dans un sens, hébergé des déracinés - ayant survécu aux calamités et à la guerre, ou rapatriés d'anciennes colonies - afin qu'ils commencent une nouvelle vie après l'expérience d'une blessure.

\section{Traduire la banlieue parisienne au Japon}

Le retentissement des émeutes survenues en France en 2005 s'est manifesté au Japon dans la réalisation, en 2012, de la traduction du roman Le petit Malik (2008) de Mabrouck Rachedi. Le titre japonais, Kôgai shônen Mariku [Malik, garçon de la banlieue], révèle d'emblée le thème central du roman : la banlieue. La traductrice, Nakajima Saori, surtout connue pour ses essais sur la vie parisienne, explique dans sa postface pourquoi elle a ainsi «traduit» le titre : Le petit Nicolas de René Goscinny, auquel Rachedi fait référence, n'est pas bien connu au Japon; elle a donc essayé de montrer que Malik est un nouveau type de garçon français, enfant d'immigrés musulmans et donc très différent du petit-bourgeois des années 1960 (qui ressemblerait plutôt à la petite-bourgeoise de La petite Non monte en nuages; Ishii, 1951). 
Les traductions, qu'elles soient littéraires ou scientifiques, sont publiées au Japon dans une optique plus ou moins «pédagogique»; elles sont toujours suivies d'une postface du traducteur et parfois d'un commentaire sur l'ouvrage, écrit par le traducteur ou par quelqu'un d'autre. La postface du Petit Malik présente d'abord le contexte historique de la banlieue de France et les conditions des ouvriers-immigrés, en les comparant au contexte et aux conditions de la banlieue japonaise dont les cités ${ }^{5}$ ont aussi hébergé les cols blancs, surtout dans les années 1960. La traductrice explique pourquoi cette «tranche de vie» étrangère mérite d'être lue au Japon :

Il y a beaucoup de gens qui ont connu «la question de la banlieue» par les émeutes survenues en France en 2005; les voitures brûlées ont été couvertes tous les jours par les médias. Après ce premier moment où le mécontentement des jeunes des banlieues a explosé, les médias n'en disent plus rien, mais la situation de ces jeunes ne s'est pas du tout améliorée. Les médias se focalisent sur un propos seulement quand il y a des affaires graves, et, une fois le feu calmé, ils n'en parlent plus. Pourtant, cette attitude n'est pas correcte. Ce que Le petit Malik nous apprend, c'est qu'il existe de graves problèmes dans les banlieues, mais que la vie quotidienne, ordinaire, continue en embrassant ces problèmes; s'il arrive une affaire ou pas, s'il arrive quelque chose de grave ou pas, s'il arrive un crime ou pas, il n'y a qu'une petite différence entre les deux résultats. [...]

Cette brillante œuvre littéraire nous permet de comprendre, en faisant appel à l'intelligence et la sensibilité des lecteurs, ce que même les études les plus minutieuses et les reportages les plus détaillés ne sauraient transmettre. Rachedi décrit de façon vivante le mécanisme selon lequel des personnes ordinaires vivant ordinairement tombent, sans se faire remarquer, dans la misère, ainsi que la psychologie du cercle vicieux où des personnes élevées dans un milieu défavorisé s'enfouissent dans leur milieu. Cependant, sa manière de peindre la banlieue ne se limite pas à la «misère» ou à la «conscience du problème», ne présente pas uniquement la «dénonciation» à voix haute ou la "colère», mais enveloppe la réalité d'un humour qui balaie tout avec un sourire. À l'ombre de cela, il y a son regard tendre envers les défavorisés et le courage de vouloir prendre la situation en charge. (Nakajima, dans Rachedi, 2012, p. 196-197; notre trad.)

5. Ces cités aujourd'hui très vieillies sont souvent habitées par des personnes âgées vivant seules et des familles étrangères défavorisées (v. Ônishi, 2017). Notons aussi qu'il y a eu récemment des cités joliment rénovées pour attirer les jeunes (v. Makihirochi, 2017). 
Présenter la banlieue sans l'expliquer - c'est-à-dire en évitant le discours universitaire ou médiatique - est un principe ambivalent pour la traduction littéraire, notamment si elle est "pédagogique»; non seulement la postface de la traductrice et le commentaire de la romancière Nakajima Kyôko, sœur de la traductrice et connaissance personnelle de l'auteur, sont ajoutés au livre, mais chaque chapitre est aussi suivi de notes de la traductrice, expliquant au lecteur japonais ce qu'est la «Nutella» ou le fonctionnement du système scolaire français. Par exemple, on compte neuf notes pour les dix pages du chapitre «Onze ans» ${ }^{6}$.

Les informations paratextuelles révèlent souvent la posture de l'éditeur et du traducteur ainsi que la manière dont ils ont voulu présenter la traduction. Le Ventre de l'Atlantique (2003) de Fatou Diome, par exemple, a été traduit en japonais en 2005 avant les émeutes -, également par une traductrice-essayiste vivant à Paris, Takahata Yûki. Comme la traductrice du Petit Malik, Takahata Yûki s'intéresse à la vie quotidienne en France et tente aussi de présenter, dans sa postface, la situation des immigrés et les rapports historiques avec les anciennes colonies africaines. Elle s'abstient toutefois d'ajouter trop de notes à ce «roman autobiographique de l'écrivaine d'origine sénégalaise» (expression en bandeau sur l'édition japonaise; notre trad.). Tous mes amis (2004) de Marie NDiaye, traduit en japonais en 2006 par Kasama Naoko, semble être présenté dans un souci plus «littéraire»: la traductrice, chercheuse spécialiste de Gustave Flaubert, a ainsi rédigé trentequatre pages de commentaire pour accompagner sa traduction et présenter son analyse de l'écriture de Ndiaye, le tout appuyé de nombreuses références. Un autre cas plutôt curieux est représenté par la traduction de Soumission de Michel Houellebecq (2015, traduit la même année au Japon). Cette traduction est en effet suivie d'un commentaire signé par un ancien diplomate, Satô Masaru, très connu comme spécialiste de politique internationale et de théologie chrétienne. Dans ce cas, il apparaît évident que l'éditeur a voulu situer le roman par rapport à l'actualité politique, en suggérant la relation avec l'attentat «islamiste» contre la rédaction de Charlie Hebdo. Notons que la traduction japonaise de 2084 la fin du monde de Boualem Sansal (2016) a été publiée en 2017 chez le

6. Nous considérons ces notes très utiles aux lecteurs japonais et estimons que les efforts de la traductrice sont énormes et précieux. 
même éditeur et que, dans les deux cas, les traductrices sont des professionnelles (ce ne sont probablement pas elles qui ont choisi de traduire ces ouvrages et de les présenter).

La difficulté de traduire la banlieue de France réside, nous semble-t-il, dans les noms propres et les realia inconnus des lecteurs japonais. Nakajima Saori avoue quelle a eu beaucoup de peine à traduire la «langue de la banlieue» notamment les argotismes, les néologismes et les gros mots, jusqu'à certains termes de football (un sport devenu récemment populaire au Japon, quoique plutôt réservé à la classe moyenne). Les énoncés intraduisibles, les realia de la société étrangère que la traductrice a voulu expliquer par des notes, n'ont pas d'équivalents en d'autres langues. Mais faut-il vraiment connaître Joe Dassin ou l'expression «bobo» (bourgeois bohème), ou savoir distinguer "Nutella» et «Mutella», afin de pouvoir savourer un roman comme Le petit Malik? En fait, ce sont la gentillesse de Bruno ou l'affection de la mère du héros qui nous touchent, et les conditions qui règnent dans les banlieues ne sont que secondaires à notre compréhension de la vie quotidienne qui y règne. Voilà l'ambivalence de la littérature de banlieue : il faut oublier ses conditions socioculturelles, quoique marquantes, pour atteindre le vif de l'expérience littéraire et éviter de la réduire au cliché sociologique. Nakajima Kyoko écrit ainsi dans son commentaire : «J'espère que l'on s'amuse en lisant cette histoire coquine, drôle, triste et poétique, sans trop penser à des choses superflues" (dans Rachedi, 2012, p. 201; notre trad.).

Les noms propres nont généralement pas de sens par euxmêmes. Ce sont des noms de choses parfois banales et apparemment interchangeables, qui ne sont cependant pas remplaçables pour ceux qui vivent dans le milieu en question, car ces noms intraduisibles (sorte de «lexique familial» ${ }^{7}$ ) sont des composants précieux de leur quotidien. En écrivant des tranches décousues de la vie quotidienne de sa famille, le dernier grand écrivain de la banlieue de Tokyo, Shôno Junzô, affirme : «Quoiquéphémères et incohérentes, s'il avait manqué même une de ces choses, j’aurais senti de l'insipidité» (cité dans Kawamoto, 2012 [2003], p. 380; notre trad.). Certes, comme Shôno n'a cessé d'écrire la «joie» de sa famille, Mabrouck Rachedi décrit, lui aussi, la vie de Malik (et de ses camarades), qui, malgré les nombreuses difficultés, n'est pas

7. Cf. Natalia Ginzburg (1963). 
malheureuse non plus. C'est là où nous trouvons une coïncidence, une ambition commune entre les deux littératures de la banlieue française et japonaise : crier le bonheur de la vie, discrètement, audelà de toutes les conditions défavorisées, soient-elles latentes ou patentes.

\section{La banlieue vue par Horie Toshiyuki}

Si un mot perd quasiment son sens premier et devient un déclic précieux pour la mémoire, il n'est plus traduisible ni interchangeable avec aucun équivalent : Oparaban [Auparavant] (Horie, 1999) nous apprend l'irremplaçabilité d'un mot précieux qui se rapproche exceptionnellement du nom propre. Cette nouvelle inoubliable d'Horie Toshiyuki (1964-), l'un des plus importants romanciers de la littérature japonaise contemporaine, raconte une rencontre éphémère avec un "professeur» (ainsi l'appelait-on) chinois dans la cité universitaire de Paris quelques années après la Révolution culturelle. Oparaban, c'est la transcription phonétique du mot français «auparavant» qui s'employait alors trop souvent chez les immigrés chinois. Le narrateur éprouve un peu d'antipathie pour les Français qui se moquent "des camarades asiatiques» (Horie, 1999, p. 13; notre trad.) avec ce «cliché» (ibid., p. 14; notre trad.), mais, une fois ce mot prononcé de la bouche d'un voisin, il en est complètement charmé. L'histoire se déroule lentement autour d'un grand joueur de ping-pong, en racontant la vie des «étrangers» qui vivent aux marges de Paris.

Pour Horie Toshiyuki, chercheur et traducteur de littérature française, la banlieue parisienne est un lieu où il peut trouver «un autre œil» pour regarder le monde différemment. C'est avec Kôgai e [Vers la banlieue] (1995) qu'il a fait ses débuts comme écrivain. Les nouvelles qui composent l'ouvrage, d'abord publiées dans la revue mensuelle Furansu ${ }^{8}$ [France] pendant douze mois, ont été interprétées comme un essai autobiographique. Dans la postface de l'édition $U$-Books (Horie, 2000), l'auteur confie qu'en réfléchissant au traitement de la banlieue dans la littérature française contemporaine, il avait voulu produire une série de textes critiques présentant de telles œuvres. Il a plutôt fini par rédiger des textes qui sont entièrement fictionnels. D'après lui, le narrateur n'est pas l'auteur, mais «seulement un porte-parole de la position "de banlieue”»(Horie, 2000, p. 190; notre trad.).

8. Revue japonaise spécialisée en langue et culture françaises publiée depuis 1925. 
«450 francs, je ne peux plus baisser le prix [...]» (Horie, 2000, p. 7; notre trad.). Le premier chapitre du livre d'Horie, « Remington portative», commence ainsi, dans le marché des antiquaires qui entoure le Square du Temple à Paris, par la négociation du prix d'une machine à écrire de marque Remington entre le narrateur et un garçon d'apparence collégienne qui garde le stand de son père. Le narrateur trouve cette machine à écrire dont la marque américaine lui a été rendue familière par le poème "Lettre» de Blaise Cendrars. Le narrateur cite des extraits de ce poème simple adressé à l'amie de l'écrivain vagabond, en le traduisant de manière naturelle et harmonieuse tout en l'intégrant au style de son propre récit. Il ajoute que malgré la banalité des expressions amoureuses contenues dans le poème, il en a été fort touché quand il a imaginé Cendrars tapant cette lettre avec sa main gauche ${ }^{9}$ sur sa Remington, dans une petite chambre d'un bateau à destination du Brésil. Cependant, il précise que c'est d'abord un portrait du poète suisse qui l'a impressionné :

Dans quelques photos qui ont été prises pendant la nuit figure Cendrars au travail. Au-dessus de la table est suspendue une ampoule munie d'un simple abat-jour [...]. Bouteille de vin, cigarettes, cendrier et encrier sont disposés près de sa main libre, et sa Remington est solennellement assise à côté de son bras droit dont la manche est trop longue. Les photos de Cendrars en train d'écrire semblent avoir été prises en rafale. En suivant leur ordre, on voit comment il corrige avec sa main gauche le manuscrit tapé avec la Remington. (Horie, 2000, p. 10-11; notre trad.)

Le narrateur, fasciné par la Remington de Cendrars, veut dès le début posséder cette antiquité qu'il vient de dénicher. Invité par le garçon à confirmer qu'elle fonctionne toujours, il a aussitôt envie de l'utiliser. Le garçon finit par proposer à son père de présenter au client japonais Angelo, un réparateur qui a ouvert un atelier en banlieue, plus précisément à Gentilly.

Avant de nous amener en banlieue, le récit particulièrement sinueux d'Horie ${ }^{10}$ se détourne vers le photographe Robert Doisneau,

9. Cendrars a perdu sa main droite pendant la Première Guerre mondiale.

10. Son style est fortement marqué par la sinuosité et la longueur excessive des phrases, qu'on observe souvent dans les textes traduits depuis des langues occidentales (surtout du français). Un tel style est parfois appelé hon'yaku-chô [ton traduit], indiquant péjorativement des écritures moins lisibles ou moins naturelles, par exemple, celles d'Ố Kenzaburo et de Murakami Haruki. 
en disant que les portraits qu'il a pris «capturent avec une magie impressionnante la part la plus intime de Cendrars» (Horie, 2000, p. 13). C'est en 1945 que Doisneau a rendu visite à l'écrivain alors retiré à Aix-en-Provence afin de prendre des photos pour la publicité de son dernier roman autobiographique, L’homme foudroyé. Ils se sont rencontrés et très bien entendus, et le poète a encouragé le photographe encore méconnu à rassembler ses photos inédites pour en faire un recueil. De fait, Cendrars aurait trouvé un éditeur, Pierre Seghers, pour publier un livre photographique de luxe en 1949. C'est le premier chef-d'œuvre de Doisneau, La banlieue de Paris (traduit en japonais en 1992).

Ainsi, le mot «banlieue» résonne doublement, par le biais de la machine à écrire de Blaise Cendrars, entre le présent du narrateur et le passé de Robert Doisneau, un demi-siècle auparavant :

En traçant les paysages de la banlieue de Paris recueillis dans ce livre photographique, on se rend compte pourquoi Doisneau a pu puiser la poésie si justement dans le quotidien de Cendrars dans le Midi. Son atelier, qui doit être l'espace le plus intime, le plus sacré, le parfum de son atelier où la Remington portable et des manuscrits noirs remplis de lettres sont aperçus; nous voyons avec nos yeux le parcours de ce parfum qui émane de l'infiltration souple et organique dans la vie quotidienne. Le temps passé dans la cuisine, où le poète manipule de la main gauche, sérieusement et si heureusement, le moulin à café, et verse le café dans un bol épais, pèse autant que le temps où il s'absorbe dans la création. Sa silhouette croisant une fille dans une rue étroite pleine de linge suspendu des fenêtres, son audacieux ventre comme un tambour, nu sur la falaise à Villefranche, son simple bavardage debout avec un aiguiseur de couteaux, et son chuchotement d'informations douteuses aux enfants gitans, tous ces moments se focalisent, par la main de Doisneau, sur l'image d'un homme pour qui tout se convertit en poésie. C'est probablement vrai qu'un objet extrêmement original comme Cendrars renforce lui-même ses photos, mais ici existe déjà le style d'un photographe habile, sachant marier technique et sensibilité, et ayant créé des œuvres qui doivent se recueillir comme La banlieue de Paris. (Horie, 2000, p. 14-15; notre trad.)

La maturité du photographe est ainsi attestée par ce talent qui lui permet de saisir immédiatement le «quotidien» nu et intime de l'objet; en même temps, c'est la quotidienneté qui marque essentiellement «les paysages de la banlieue» photographiés par Doisneau. 


\section{Histoire d'une transcription}

En racontant ainsi Robert Doisneau, Horie Toshiyuki détache la «banlieue» du vocabulaire standard et présente ce mot comme un nom propre français en le doublant d'un surtitre (en ruby, petites lettres employées pour surtitrer). La «banlieue» se traduit en japonais kôgai qui s'écrit 郊外 en kanji (lettres d'origine chinoise). Ces idéogrammes peuvent être surtitrés en kana, lettres phonétiques japonaises, pour indiquer leur prononciation (surtout pour les lettres rares et difficiles à lire; celles des livres pour enfants et des mangas sont aussi surtitrés), et cette technique de dédoublement peut être employée aussi pour la description bilingue. Cette transcription japonaise très originale, auparavant employée pour expliquer les mots dérivés de l'ancien chinois, peut circonscrire plus précisément le signifié d'un mot ou créer quelque effet de distanciation avec l'original phonétique étranger transcrit en kana. C'est le lauréat du prix Nobel de littérature Ố Kenzaburô (1935-), entre autres, qui l'a utilisée afin d'explorer de nouveaux effets littéraires dans ses romans.

Nous ne savons pas quand et qui a commencé à appliquer cette transcription au mot "banlieue», mais maintenant, grâce à cette écriture bilingue, beaucoup d'intellectuels japonais non francophones savent que banryî est un nom propre indiquant la banlieue de France, qui est ainsi bien distinguée des autres banlieues du monde entier. En retour, le mot japonais kôgai a fini par acquérir quelque nuance parisienne, car la banlieue de Paris est, semble$\mathrm{t}$-il, devenue la représentante des banlieues étrangères au Japon. En fait, la mutation du sens avait peut-être commencé beaucoup plus tôt. Le mot kôgai est une sorte de mot-valise composé de kô et gai. À l'origine, kô indiquait le faubourg ou le «extra muros», ce qui veut dire que ce mot lui-même suffisait à l'origine, en ancien chinois, à indiquer la banlieue. Mais, en le transformant en un mot polysyllabique, plus usuel dans le langage courant, on ajouta un autre mot qui indiquait «le dehors», et les Japonais adoptèrent ce mot-valise doublant le sens de «dehors» et de "hors des murs». En effet, les Chinois préfèrent un autre mot, kôku 郊区 (jiaoqu en prononciation pékinoise), qui se traduirait par «arrondissement du faubourg». L'étymologie n'est bien sûr pas très importante ici, mais il faut reconnaître la présence ineffaçable de cet idéogramme gai 外, par lequel nous recevons inconsciemment l'impression assez forte $\mathrm{du}$ «dehors». Revenons au texte d'Horie, qui explique effectivement le terme français par son étymologie : 
Le mot français «banlieue/banrŷ̀», qui désigne aujourd'hui principalement les régions situées en périphérie de Paris, signifiait à l'origine l'étendue d'environ «une lieue/ryû » hors des murs de laquelle le seigneur exerçait le «ban/ban». Théoriquement, on pourrait dire que l'on entrerait, soit de près soit de loin, dans le territoire appelé banlieue en sortant d'un pas des murs de Paris. C'est avec la sensation de mutation côtoyée par ce pas précieux que Jacques Réda, en suivant le courant de Léon-Paul Fargue appelé "promeneur de Paris ", élève la promenade vers la banlieue en acte actif invitant au domaine de la rêverie $\left[m u s o{ }^{11}\right.$ et de la création, à l'origine de son recueil poétique Hors les murs dont le titre est, à lui seul, assez évocateur. De fait, aux portes ouvertes dans les murs entourant Paris, il y a souvent les arrêts de bus en destination de la banlieue et, si on prend l'une de ces lignes avec trois numéros, on verra aussitôt s'étendre un espace étranger au centre-ville de Paris. L'apparence du ciel, la vue des bâtiments, les allures des gens, leurs visages et cætera, varient de façon nuancée mais assurée. Quant à la définition la plus simple et la plus précise du mot ambigu «banlieue», ne serait-ce pas «hors des murs» en tout cas, quoique chaque banlieue forme son propre monde d'après les conditions de son lieu et change sa figure au fil du temps? Si je réfléchis sur ma propre expérience qui, de temps en temps, charmé par le diable, a orienté mes pieds vers le dehors de Paris et m'a porté à prendre des bus sans aucune destination ou à continuer de marcher jusqu'à ce que la fatigue commence à envahir mon corps, le sens de distance du mot banlieue me semble se rattacher aisément à celui de l'ancienne unité japonaise «ri» [里] qui se conforme aux souffles des ceux qui marchent. (Horie, 2000, p. 15-16; notre trad.)

L'idéogramme 里 fut, à l'origine, une unité regroupant cent familles en Chine antique, mais il s'employa également comme unité de longueur qui mesurait, au Japon, «trois cent pas » au VIII siècle, et environ quatre kilomètres depuis le $\mathrm{XVII}$ e siècle. Cette unité, désuète en japonais contemporain, est parfois ainsi employée pour traduire des unités de longueur étrangères. En profitant de cette coïncidence des lettres, les deux mots indiquant la longueur «lieue/ ri» s'associent, par force, dans l'imagination de l'auteur. Cette rhétorique nous semble peu convaincante, mais elle est révélatrice du point de vue d'un promeneur qui veut saisir l'espace en mutation par son corps sensible.

11. Ce terme fort littéraire pourrait paraître un peu «snob» par l'évocation de Jean-Jacques Rousseau, car il est, comme titre d'un livre, presque exclusivement employé pour traduire Les «rêveries» du promeneur solitaire. 


\section{Toucher la "position » de la banlieue}

La posture de promeneur d'Horie Toshiyuki semble succèder à celle du «flâneur» de Paris de Walter Benjamin, surtout quand l'auteur prononce en allemand le mot-clé du penseur, "Aura» (Horie, 2000, p. 11), ou à celle de Jean-Jacques Rousseau quand il raconte ses «rêveries de promeneur» (ibid., p. 109; notre trad.). Cependant, si nous considérons l'histoire littéraire de la banlieue de Tokyo, la promenade en observant les paysages est, en fait, une pratique remontant jusqu'à Doppo et Tourgueniev (notons que Shonô Junzô, lui aussi, s'est promené tous les jours et en a rendu compte dans ses dernières autofictions).

Horie Toshiyuki remarque que «les corps» de Blaise Cendrars et Robert Doisneau connaissaient aussi bien l'un que l'autre la banlieue parisienne, même s'ils l'ont vécue différemment. Les photos à publier que Cendrars a sélectionné dans le Midi ont été prises entre 1947-1949 par Doisneau, mais ce dernier se souvenait en réalité de la banlieue de l'entre-deux-guerres, en se plongeant dans les images recueillies pendant l'après-guerre :

Non seulement les temps ne concordent pas, mais aussi, comme Cendrars le déclare dans le texte, les deux n’ont jamais marché ensemble dans la banlieue. Le texte de Cendrars ne se limite pas à être un commentaire des photos de Doisneau; il est écrit comme une œuvre indépendante, qui n'est pas un résultat mené par des expériences visuelles en commun, mais un produit de la réaction de la mémoire et du langage de l'un, face aux images fixées sur papier photographique par l'autre. (Horie, 2000, p. 16; notre trad.)

C'est donc une association anachronique de mémoires visuelles et visionnaires qui est à la base du livre photographique réalisé en collaboration avec le poète. De même, la nouvelle d'Horie (comme toutes les autres dans le même recueil) est tissée par des paysages de banlieue pour le narrateur-promeneur comme nous en avons déjà cité, ainsi que par plusieurs références à des œuvres littéraires et photographiques. Il semble que l'auteur reconnaisse en Cendrars une figure typique du visionnaire qui découvre en images une sorte de profondeur historique, tandis que Doisneau, "né à Gentilly", veut peut-être priver les images saisies dans l'immédiateté de toutes les histoires qu'elles pourraient conserver.

Si Cendrars veut lire des histoires pittoresques, analogues aux scènes de voyages au Brésil ou en Russie, derrière le dos des gens de banlieue 
recherchant dans les compétitions de cyclisme leur rare amusement, Doisneau, de son côté, veut simplement transmettre une chose à laquelle sa rétine a instantanément réagi. Même si la vie présentée en images aurait été celle de son pays natal pour lequel il éprouvait un grand attachement. (Horie, 2000, p. 19; notre trad.)

Après ce long détour, le narrateur se rend enfin à Gentilly, mais il ne peut découvrir dans le paysage aucune histoire pittoresque, et il se rappelle les phrases de Doisneau qui se plaint du quartier devenu trop fonctionnel pour être beau. Les histoires du quartier sont maintenant invisibles à l'oeil :

Le numéro que j'ai trouvé après avoir repéré sur la carte l'adresse et parcouru le quartier pendant un moment, correspondait à un appartement sans goût avec une grande salle d'entrée dans une rue qui ne se faisait pas remarquer visuellement, à moins que l'on n'eût quelques connaissances de l'histoire de la ville. (Horie, 2000, p. 21; notre trad.)

Dans le discours du narrateur, la question de l'historicité de la banlieue reste ambiguë: devons-nous en explorer ses histoires, soit révélées soit dissimulées, à travers la mémoire ou le paysage du quartier? Le narrateur ne s'attarde jamais sur les histoires de chaque quartier de banlieue : il se limite à en caresser le paysage de façon sensible en se plongeant dans les fragments littéraires qui le racontent. Il représente dans son récit une figure sans "contours", une "position" de la banlieue sans l'expliquer sociologiquement ni la réduire aux éclats de mémoires livresques :

L'espace où l'arrière-plan qui montre les humains vivants est essuyé. La pauvreté que porte la banlieue contemporaine est différente de celle d'autrefois en phases comme en dimensions, tandis que le phénomène de banlieuisation que Cendrars a sévèrement dénigré dans La banlieue de Paris doit être le problème commun, non seulement pour Gentilly, mais aussi, à la fin du XX siècle, pour toute zone qui s'étend en dehors de Paris. Malgré cela, il ne serait pas impossible que de ces ruines en béton naissent un nouveau Doisneau, un nouveau Cendrars. Non, il y a plus de possibilités que de "l'arrière-plan inhumain » naisse paradoxalement «l'arrière-plan humain». (Horie, 2000, p. 20-21; notre trad.)

La nouvelle se clôt par un murmure du réparateur qui a trouvé une adresse perdue de Paris dans la plaque de la Remington: «natsukashii na» (Horie, 2000, p.21; notre trad.). Cet énoncé 
presque intraduisible qui dénote l'émotion nostalgique nous semble être une expression très japonaise. Elle ferait allusion au caractère fictif du récit qui a été créé dès le début en japonais, mais qui se lit comme un essai autobiographique "vécu» en français.

\section{En guise de conclusion}

La banlieue est, comme nous l'avons vu, un lieu en mutation, un espace où les défis sociaux s'entassent coup sur coup et dont les sociologues s'enquièrent, pour le présent comme pour l'avenir; un territoire où les paysages autour des habitations changent et où les écrivains découvrent de nouvelles esthétiques, où les vies du peuple perdurent dans des difficultés et où le bonheur côtoie la misère. Grâce à la traduction des littératures occidentales, la littérature japonaise a découvert de nouvelles valeurs dans la banlieue de Tokyo : la promenade dans les bois, basée sur une nouvelle sensibilité envers la nature, est inspirée par Tourgueniev, et l'image des enfants des classes moyennes entourés de soins familiaux nous vient de la littérature anglaise pour enfants. À cela il faut ajouter le travail des traductrices japonaises de la littérature qui aborde le sujet de la banlieue française contemporaine; ces traductrices tentent, d'un côté, de présenter les souffles de ses habitants que les recherches scientifiques tout comme les approches médiatiques ne sauraient saisir, et, de l'autre, ne peuvent se dérober à l'emploi de notes pour aider les lecteurs à comprendre le contexte sociologique des œuvres. Horie Toshiyuki, qui a transposé la banlieue de Paris directement dans la littérature japonaise, semble, pour sa part, peu favorable à une approche sociologique :

Il n'y a rien en banlieue. Cela peut être vrai. Pourtant, afin de savoir d'où vient ce sentiment de ne rien avoir, pourquoi est né le quartier dévergondé où les mauvais garçons traînent, ce qui est important n'est pas, par exemple, de mobiliser plusieurs spécialistes au nom du principe de la sociologie pour réaliser des enquêtes de grande envergure, mais de faire fonctionner d'abord ses propres pieds et yeux dans l'espace cru. Fixer les yeux sur le changement passif du quartier en banlieue mène à regarder le passé et le présent de soi-même, et la vie apparemment ordinaire et le passé abominable collé à son envers sont laissés intacts au fond du cœur, comme une sensation réelle qui ne peut être substituée ni ordonnée par paraboles adroites ou sentimentalisme de pacotille, ce qui crée la vigueur exceptionnelle de ce livre. (Horie, 1990, p. 91-92; notre trad.) 
Horie écrit que cette chronique du voyage de Maspero et Anaïk Frantz est un «fruit de la pensée précieuse qui donne un coup sévère à ceux qui ne veulent pas trouver des histoires qu'à l'intérieur "des murs" (Horie, 2000, p. 81-82; notre trad.). La banlieue est donc pour le narrateur une expérience du «dehors»; il sort pour découvrir des histoires cachées hors les murs. Cependant, il ne vise pas à expliquer la banlieue avec ces histoires retrouvées, mais veut, simplement, les laisser éclater autour d'elle à travers des fragments littéraires; la banlieue comme espace en mutation ne se réduit pas aux mémoires de son passé, mais se mêle toujours avec ses paysages pour être sensible à ceux qui s'y promènent.

Traduire est un désir de transmission. Faut-il alors transmettre l'objet par soi-même ou le faire comprendre par la paraphrase ou l'annotation? Le dilemme est d'autant plus complexe lorsque l'objet «banlieue» est inconnu et étranger. Kôgai e [Vers la banlieue] d'Horie Toshiyuki est conçu, selon l'auteur, pour représenter la «position» (2000, p. 190; notre trad.) de la banlieue parisienne, tandis que la traduction du Petit Malik de Mabrouck Rachedi est réalisée pour transmettre les voix de la banlieue. Rachedi rend compte pleinement des conditions socioculturelles de la banlieue dans son ouvrage, et cette tendance est accentuée par la traduction annotée. Horie, en revanche, laisse tomber la connotation sociologique en traduisant la banlieue de Paris dans les impressions de paysages procurées par les détours livresques. Cette traduction de la banlieue réalise, en fait, l'association des images, des visions, des idées des deux cultures : par exemple, quand Horie explique la mesure des pièces des petites maisons de banlieue en disant «l'espace de six tatamis et quatre et demi tatamis avec un petit eau courant [deux pièces et une cuisine]»(2000, p. 107; notre trad.), il traduit l'espace d'habitation avec l'unité de longueur japonaise traditionnelle, la natte de paille. Le narrateur se souvient ensuite du livre d'images de Virginia Lee Burton, The Little House (1942), dont il a lu, enfant, la traduction par Ishii Momoko (1954). En profitant de choses connues pour comprendre des choses inconnues, les images s'associent pour générer des paysages qui font pressentir le surgissement de l'inattendu.

Létrangeté des nouvelles d'Horie autour de la banlieue parisienne vient $\mathrm{du}$ fait qu'elles sont remplies de noms propres peu connus : noms de lieux parisiens et noms d'écrivains et de photographes français, dont la plupart sont étrangers aux lecteurs 
japonais. Le narrateur, pourtant, ne les explique guère; il les laisse flotter comme l'air qui remplit les paysages de banlieue. Il évite ainsi d'accorder des significations définitives à ces quartiers, en profitant des mémoires et des histoires concernant ces noms. Son étrange réserve se remarque lorsqu'il déclare qu'il lit toujours la traduction en français des littératures étrangères pour se détendre, pour lire aisément sans oser connaître la langue d'origine (Horie, 2000, p. 61-63). La banlieue demeure ainsi un lieu en devenir, où les promeneurs s'égarent dans leurs rêveries, et la traduction demeure, pour sa part, un lieu de partage où le sens est suspendu et remis en question.

\section{Références}

Begag, Azouz (1986). Le gone du Chaâba. Paris, Seuil.

Lee Burton, Virginia (1942). The Little House, Boston, Houghton Mifflin.

Lee Burton, Virginia (1954). Chiisai ouchi [The Little House]. Trad. Ishii Momoko. Tokyo, Iwanami Shoten.

Cendrars, Blaise (1945). L'homme foudroyé. Paris, Denoël.

Diome, Fatou (2003). Le Ventre de l'Atlantique. Paris, Éd. Anne Carrière.

Diome, Fatou (2005). Taiseiyô no kaisô no youni [Le Ventre de l'Atlantique]. Trad. Takahata Yûki. Tokyo, Kawadeshobô Shinsha.

Doisneau, Robert (1949). La banlieue de Paris. Paris, Seghers.

Doisneau, Robert (1992). Pari Kôgai [La banlieue de Paris]. Tokyo, Riburopôto.

Futabatei, Shimei (1951 [1887]). Ukigumo [Nuages flottants]. Tokyo, Kadokawa Shoten.

Ginzburg, Natalia (1963). Lessico famigliare [Les mots de la tribu]. Torino, Einaudi.

Hasegawa, Machiko (1997-1998 [1946-1974]). Sazae-san. 23 vols. Tokyo, Asahi Shinbunsha.

Hayashi, Mizue (1984). Furansu no Ihôjin [Les étrangers à Paris]. Tokyo, Chûôkôronsha.

Horie, Toshiyuki (2009 [1998]). Oparaban [Auparavant]. Tokyo, Shinchôsha.

Horie, Toshiyuki (2000 [1995]). Kôgai e [Vers la banlieue]. Tokyo, Hakusuisha.

Houellebecq, Michel (2015). Fukujû [Soumission]. Trad. Ôtsuka Momo. Tokyo, Kawade Shobô Shinsha.

Ishii, Momoko (1967 [1951]). Non-chan kumo ni noru [La petite Non monte en nuages]. Tokyo, Fukuinkan Shoten.

Kawamoto, Saburô (2012 [2003]). Kôgai no bungakushi [L'histoire littéraire de banlieue]. Tokyo, Iwanami Shoten. 
Kunikida, Doppo (1949 [1901]) Musashino [Champs de Musashi]. Tokyo, Shinchôsha.

Jolivet, Muriel (2003). Imin to gendai furansu [Les immigrés et la France contemporaine]. Tokyo, Shûeisha.

Makihirochi (2017). Kichijôji dakega sumitai machi desuka? [Kichijôji est-elle la seule ville que vous voulez habiter?] vol. 5. Tokyo, Kôdansha.

Maspero, François (1990). Les passagers du Roissy-Express. Paris, Seuil.

Miyajima, Takashi (2014). Tabunka dearukoto toha [Ce quêtre multiculturel]. Tokyo, Iwanami Shoten.

Miyazaki, Hayao (2014[1988]). Tonari no Totoro [Mon voisin Totoro]. Studio Ghibli.

Mori, Chikako (2016). Haijo to teikô no kôgai [Exclusion et résistance dans les banlieues : les quartiers populaires des «immigrés» en France, formation et transformations]. Tokyo, Tokyo-daigaku Shuppankai.

Ndiaye, Marie (2004). Tous mes amis. Paris, Les Editions de Minuit.

Ndiaye, Marie (2006). Minna Tomodachi [Tous mes amis]. Trad. Kasama Naoko. Tokyo, Inscript.

Ônishi, Norimitsu (2017). "A Generation in Japan Faces a Lonely Death”. The Nerw York Times. 30 novembre. Disponible à <https://www. nytimes.com/2017/11/30/world/asia/japan-lonely-deaths-the-end. html> [consulté le 29 juillet 2018].

Rachedi, Mabrouck (2008). Le petit Malik. Paris, Jean-Claude Lattès.

Rachedi, Mabrouck (2012). Kôgai shônen Mariku [Malik, garçon de la banlieue]. Trad. Nakajima Saori. Tokyo, Shûeisha.

Sansal, Boualem (2015). 2084. La fin du monde. Paris, Gallimard.

Sansal, Boualem (2017). 2084 sekai no owari [2084 la fin du monde]. Tokyo, Kawade Shobô Shinsha.

Satô, Haruo (1992 [1917]). Supein-ken no ie [La Maison de l'épagneul]. Tokyo, Iwanami Shoten.

Shôno, Junzô (2014 [1956]). Zabon no hana [La fleur de pamplemoussier]. Tokyo, Kôdansha.

Tourgueniev, Ivan (1981 [1852]). Aibiki [Mémoires d'un chasseur (1981 [1852]). Trad. Futabatei Shimei. Tokyo, Iwanami Shoten.

Satoshi Udo Département des sciences humaines Université de Kagoshima Kagoshima, Japon udosatoshi@icloud.com 